\title{
P182
}

\section{Estimation of Azimuthal Anisotropy from VSP Data Using Multicomponent Velocity Analysis}

\author{
R. Pevzner* (Curtin University of Technology), B. Gurevich (Curtin \\ University of Technology) \& G. Duncan (BHP Billiton)
}

\section{SUMMARY}

Observation of azimuthal shear wave anisotropy can be useful for characterisation of fractures or stress field. Shear wave anisotropy is often estimated by measuring splitting of individual shear-wave events on VSP data; however this method may become unreliable for zero-offset (marine) VSP where the seismogram often contains no strong individual shear events but many low-amplitude PS conversions. In this paper we introduce a new approach to estimation of fast and slow shear wave velocities and orientation of polarization planes based on the multi-component velocity analysis. This technique is applicable to zero-offset VSP data and should take advantage of the presence of a large number of shear wave events with the same velocity. The main idea is to estimate the velocity for a given polarization direction by measuring the coherency of the seismic signal of a large number of events as a function of the apparent velocity. The algorithm was tested on marine 3C VSP acquired in the North-West Shelf of Australia. These tests show good agreement between anisotropy parameters (magnitude and orientation) derived from the VSP and cross-dipole sonic log data. 


\section{Introduction}

Observation of azimuthal shear wave anisotropy can be used for characterisation of fractures (Horne, S., 2003) or stress field (Johnson and Rasolofosaon, 1996; Turner and Hearn, 1995). Shear wave anisotropy is often estimated by measuring shear wave splitting (Alford, 1986; Crampin, 1985), (Figure. 1) on VSP data. Several techniques of VSP data acquisition and analysis were suggested for these purposes (Turner and Hearn, 1995); most of them are based on measuring splitting of individual
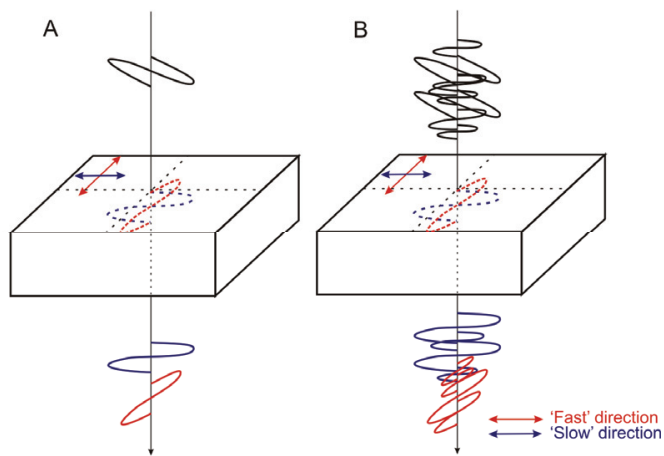

Figure 1: Shear wave splitting due to propagation through azimuthally anisotropic media phenomena shear-wave events on VSP data. These analyses involve measurement of the increase of the time delay between fast and slow shear waves with the depth (Figure 1A); it is particularly effective if the data is acquired with a shear-wave source. However, in offshore zero-offset setting, where all shear waves are converted PS events, interference between many events and generally lower shear wave amplitudes makes the analysis of time delay of individual events difficult and unreliable (Figure 1B).

In this paper we introduce an alternative approach to robust estimation of fast and slow shear wave velocities and orientation of polarisation planes, based on multi-component velocity analysis. This technique is applicable to zero-offset VSP data and should take benefit from presence of a large number

of interfering shear waves.

\section{Multi-component velocity analysis}

Our approach is to apply technique similar to standard velocity analysis, well known from CDP data processing, to traces for a given depth interval on horizontal components of 3C VSP seismogram. The main idea is to estimate the velocity of a large number of events as a function of polarisation azimuth. This is done by computing the overall coherency of all the events on a seismogram as a function of the polarisation azimuth and velocity (slope in time-depth domain). General data analysis workflow is schematically shown in Figure 2 and consists of the following steps:

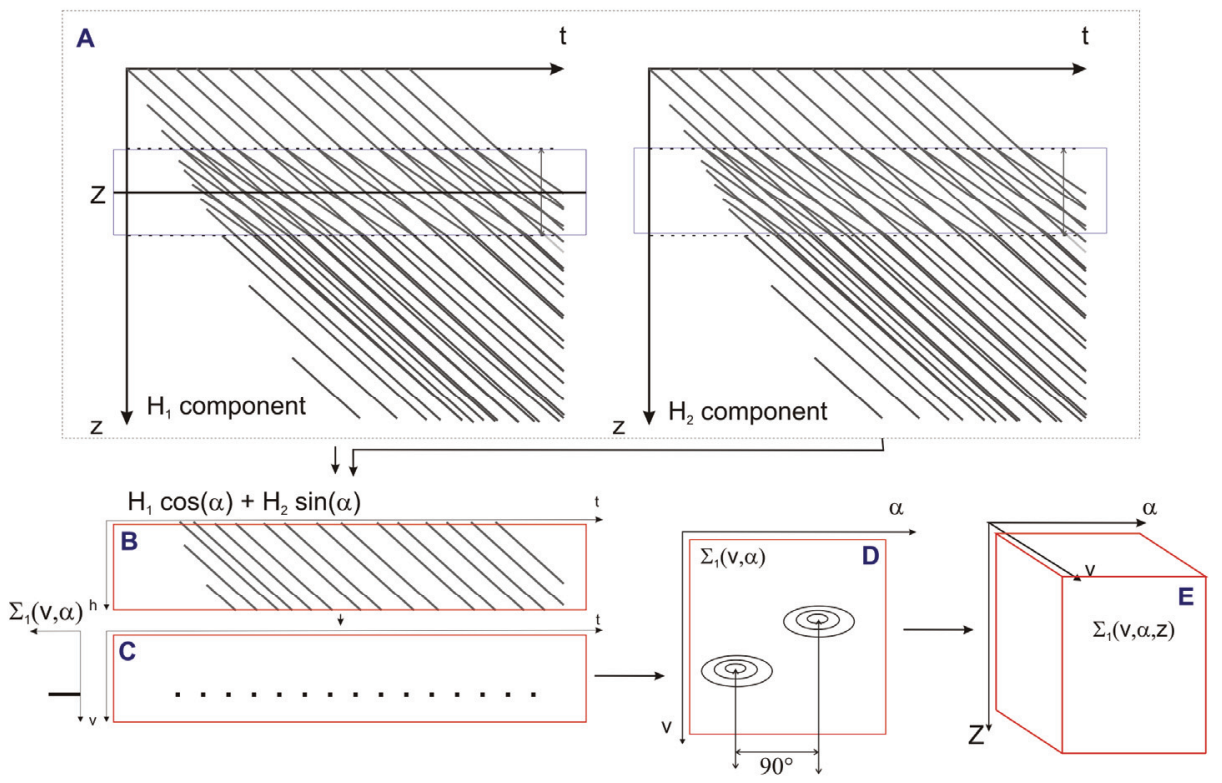

Figure 2: Principal workflow of multi-component velocity analysis 


\section{Amsterdam 'og}

1. Seismograms of the horizontal components $H_{1}(t, z)$ and $H_{2}(t, z)$ belonging to a certain depth interval are selected (Figure 2A) ( $t$ is time, and $z$ is receiver depth).

2. For the whole range $\left(0-180^{\circ}\right)$ of azimuths of polarisation, horizontal seismic 'rotated' amplitude $H(\alpha, t, z)$ as a function of azimuth $\alpha$ is computed (Figure 2B) using the equation:

$$
H(\alpha, t, z)=H_{1}(t, z) \cos (\alpha)+H_{2}(t, z) \sin (\alpha),
$$

3. To determine apparent velocities as a function of azimuth, we need to compute the velocity spectrum in a chosen depth interval (Figure 2C). This can be done by computing the coherency of the seismic signal along a linear travel-time line $t=t_{0}+\Delta z / v$, where $t_{0}$ is a reference time, $\Delta z$ is distance from the edge of depth interval and $v$ is apparent velocity. Note that this is different from the NMO velocity analysis, where traveltime curves are hyperbolas.

NMO velocity analysis is usually performed using semblance coherency measure. However semblance is not particularly suitable for our purposes since it does not take into account energy of events. If a coherent event with certain apparent velocity and polarised in certain plane exists, it will have equal impact on velocity spectrum computed for any azimuth, except for the one orthogonal to the polarisation plane. To emphasise stronger events, we propose the following modified semblance function:

$$
C=\left(\sum_{j=1}^{N}\left(\sum_{i=1}^{M} D_{i, j}\right)^{4}\right) /\left(M \sum_{j=1}^{N} \sum_{i=1}^{M} D_{i, j}^{2}\right),
$$

where $\boldsymbol{D}_{\boldsymbol{i} j}=\boldsymbol{H}\left(\boldsymbol{\alpha}, \boldsymbol{t}_{\mathbf{0}}+\Delta \boldsymbol{z}_{\boldsymbol{i}} / \boldsymbol{v}+(\boldsymbol{j}-\boldsymbol{N} / \mathbf{2}) \Delta \boldsymbol{t}, \Delta \mathbf{z}_{\boldsymbol{i}}\right)$ is a $j$-th sample of $N$ samples window on $i$-th trace along the travel time curve (after rotation), $M$ is the number of traces in depth interval being analysed. This formula differs from the semblance function by $4^{\text {th }}$ power in the numerator, which gives larger value for stronger events.

4. Computed velocity spectrum has to be stacked along the time axis (by scanning a range of $t_{0}$ values) to determine dominant apparent velocity of many events (Figure $2 \mathrm{C}, \mathrm{D}$ ). If there are two sets of coherent events representing fast and slow shear wave in a given depth interval, this stacked 'azimuthal velocity spectrum' as a function of apparent velocity and azimuth of polarisation will have two distinguished maxima, separated by $90^{\circ}$ along the azimuth axis (Figure 2D).

5. By running this analysis in a sliding window along the VSP observation interval, we'll obtain 3D volume $\sum_{\mathbf{1}}(\boldsymbol{v}, \boldsymbol{a}, \boldsymbol{z})$, (Figure $2 \mathrm{E}$ ). Interactive picking of corresponding extrema on depth slices gives fast and slow shear wave velocities and azimuths.

\section{Synthetic example}

To test the proposed approach, a simple synthetic VSP seismogram was generated (Figure 3A). On seismograms only two orthogonally polarised events are present with slightly different velocities $(1.51$ and $1.44 \mathrm{~km} / \mathrm{s})$. Figure 3B shows the result of the multicomponent velocity analysis carried out on this VSP synthetic data (length of the sliding depth window was $200 \mathrm{~m}$, one depth slice presented). One can conclude that both velocities $(1.51$ and $1.44 \mathrm{~km} / \mathrm{s})$ and polarisation planes of the events can be reliably determined from positions of the maxima of azimuthal velocity spectra.

\section{Field example from North-West Shelf, Australia}

We applied the developed algorithm to a marine rig VSP ( $\sim 50 \mathrm{~m}$ source offset, vertical borehole) dataset acquired in the North-West Shelf of Australia. Strong azimuthal anisotropy was previously reported for this area (Hung et al., 2006). Prior to the analysis, the VSP data were pre-processed by applying orientation of horizontal components, amplitude correction, bandpass filtering, and suppression of downgoing P waves. The pre-processed seismograms are shown in Figure 4. 


\section{Amsterdam 'og}
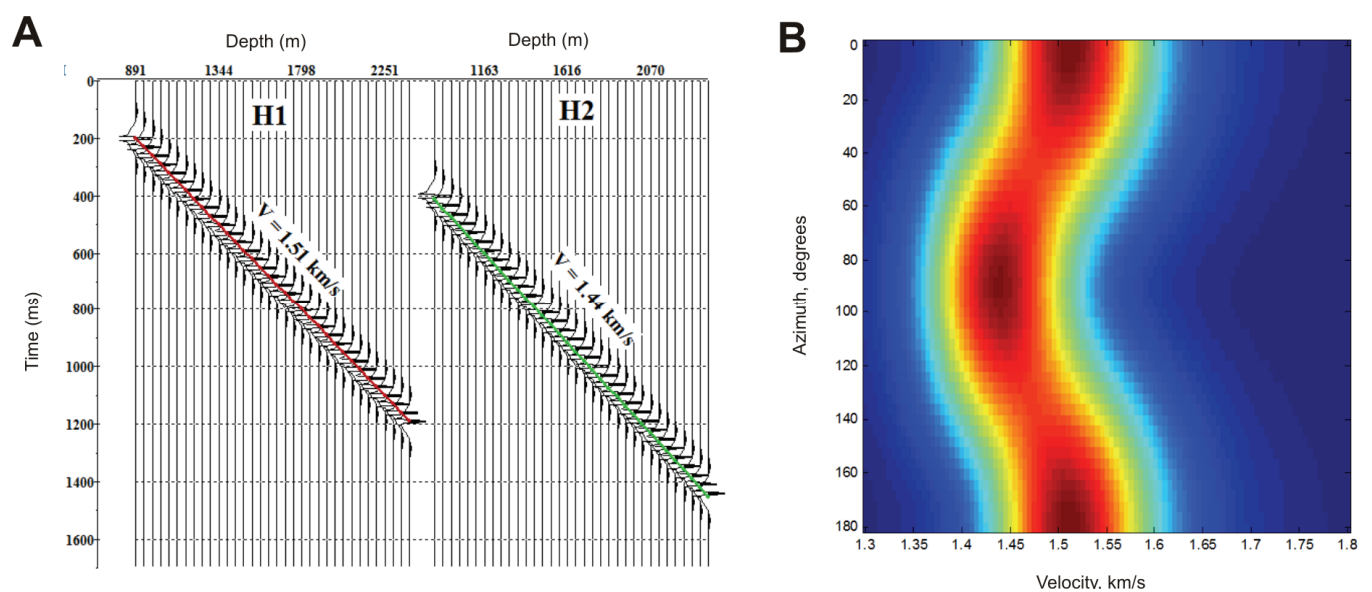

Figure 3: Synthetic example, A - horizontal components of VSP seismogram, B - azimuthal velocity spectrum.

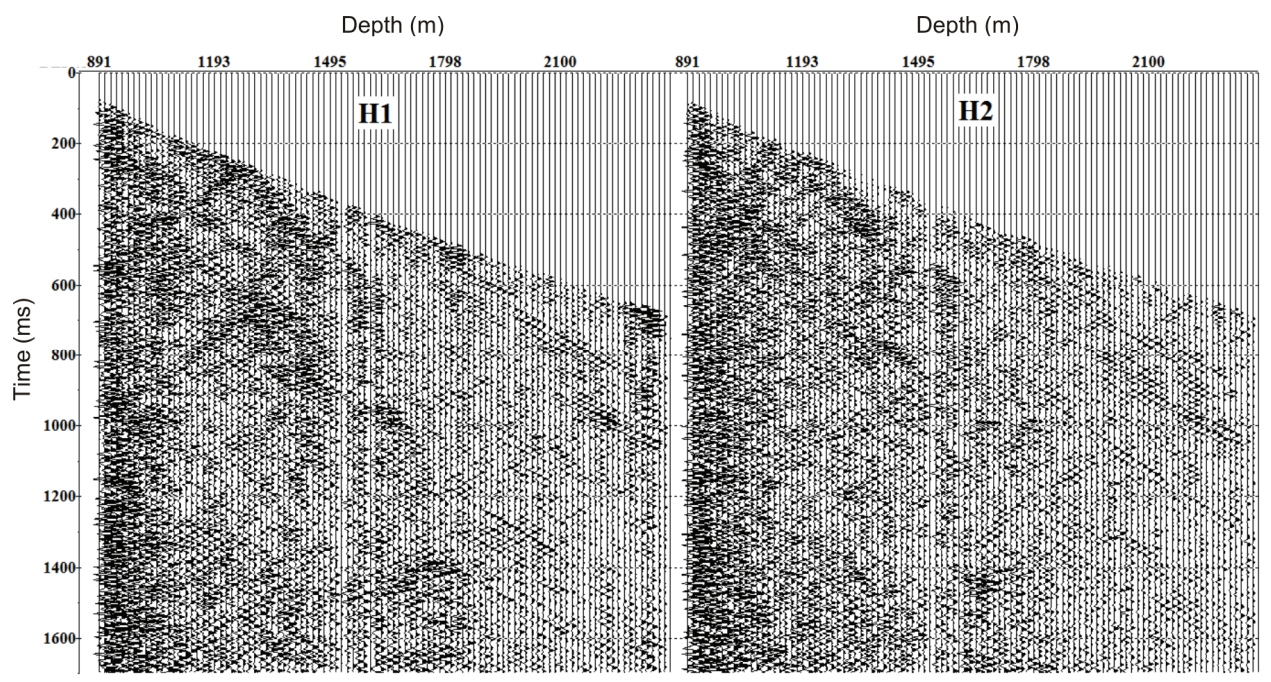

Figure 4: Real VSP data example, horizontal components after pre-processing

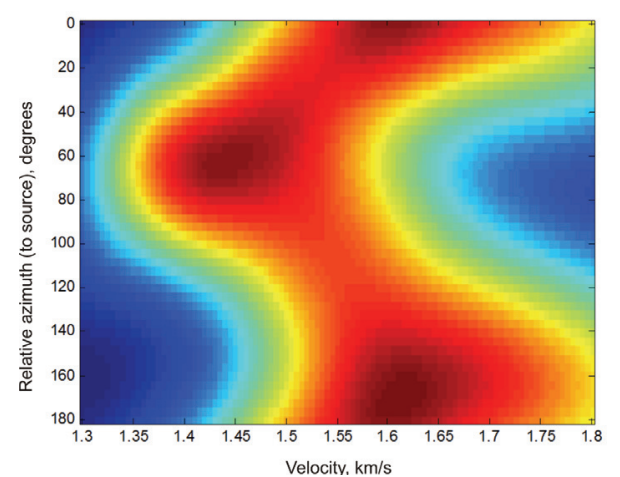

Figure 5: Example of azimuthal velocity spectrums, depth $1690 \mathrm{~m}$, time gate 2000$4500 \mathrm{~ms}$
Azimuthal anisotropy parameters were analyzed in $200 \mathrm{~m}$ length sliding window. Two different time gates (1500-2500 and 2000-4500 ms) were used to distinguish between downgoing shear waves and other events with close apparent velocities. An example of azimuthal velocity spectrum is presented in Figure 5.

The results where compared to cross-dipole sonic $\log$ measurements (Figure 6). Good agreement between these data can be observed. However, velocities derived from VSP data are slightly higher than those obtained from sonic. The azimuth of polarization of the fast shear wave from the VSP agrees very well with the one from the sonic log. 


\section{Amsterdam 'o9}

\section{Conclusions}

Yet another robust approach to estimate shear wave azimuthal anisotropy from 3C VSP data analysis is suggested. It can be applied to zero-offset VSP data obtained with standard acquisition techniques even in the marine environment. Real data tests show good agreement between anisotropy parameters derived from VSP and from cross-dipole sonic log data.
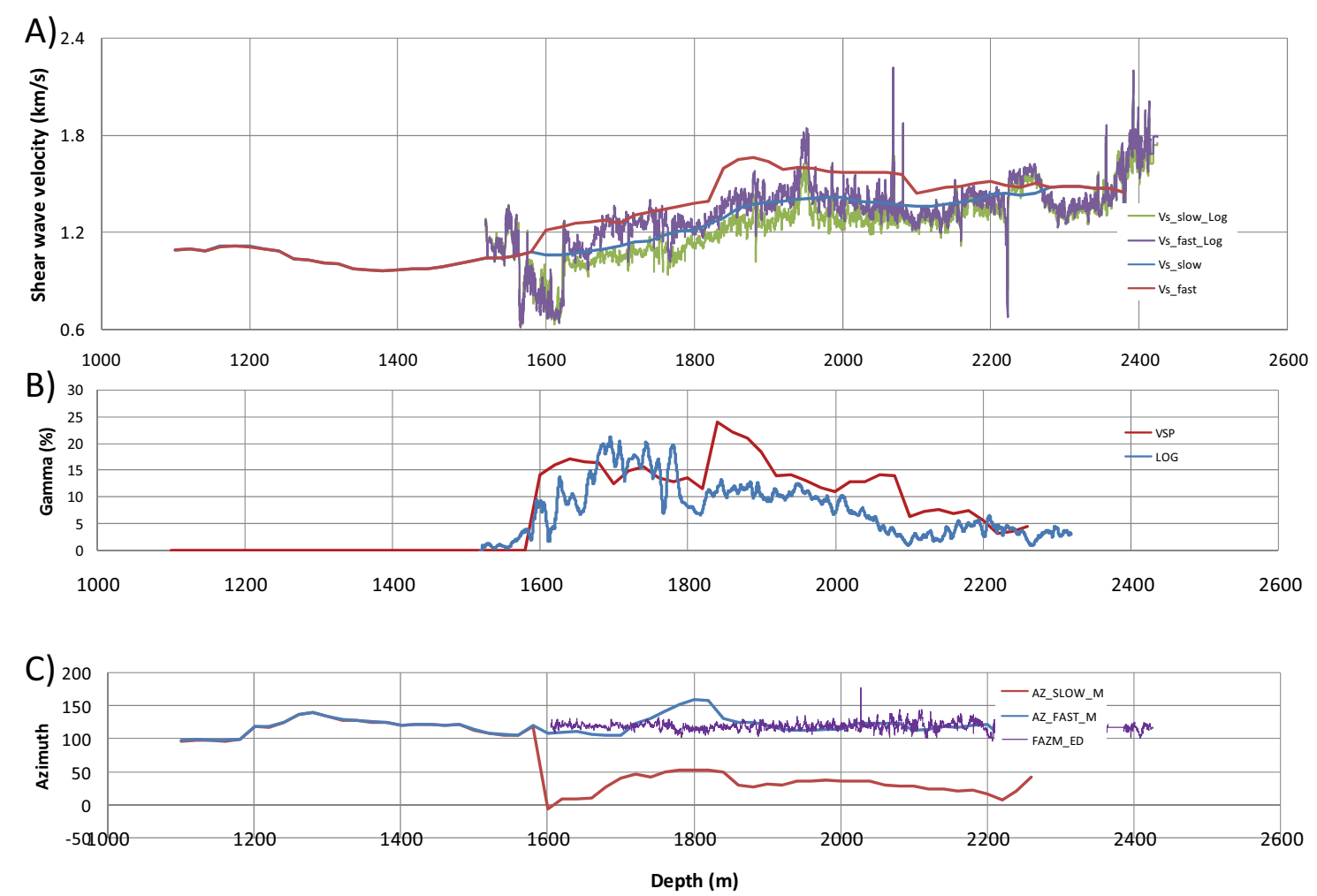

Figure 6: Comparison between fast and slow shear wave velocities (A), Thomsen r parameters (B) and azimuths of polarisation planes $(C)$ determined from VSP and cross-dipole sonic log data.

\section{Acknowledgments}

We would like to acknowledge BHP Billiton for permission to publish VSP data. The financial support of BHP Billiton and the sponsors of Curtin Reservoir Geophysics Consortium is also gratefully acknowledged. We are very grateful to Milovan Urosevic, Andrey Bona and Dariush Nardi for useful advice.

\section{References}

Alford, R. M. [1986] Shear data in the presence of azimuthal anisotropy. 56 ${ }^{\text {th }}$ SEG Annual International Meeting, Extended Abstracts, S9.6.

Crampin, S. [1985] Evaluation of anisotropy by shear-wave splitting. Geophysics, 50, 142-152.

Horne, S. [2003] Fracture characterization from walkaround VSPs. Geophysical Prospecting, 51, 493-499.

Hung, B., Zhang, F.M., Sun, J., Stanley, M., Osadchuk, A. [2006] An Automated 3D Method for Azimuthal Anisotropy Analysis in Marine Seismic Data. 68th EAGE Conference \& Exhibition, Extended Abstracts, H035.

Johnson, P.A. and Rasolofosaon, P.N.J. [1996] Nonlinear elasticity and stress-induced anisotropy in rock. Journal of Geophysical Research, 101, 3113-3124.

Turner, B. and Hearn, S. [1995] Shear-wave splitting analysis using a single-source VSP in the Otway Basin. Exploration Geophysics, 26, 519-526. 